\title{
Optimization of Biodegradable Nerve Conduit Diameter for Nerve Regeneration
}

\author{
Yoshio Ueda, Yu Sueyoshi, Takuya Nakagoshi, Noritaka Isogai* and Hirohisa Kusuhara \\ Department of Plastic and Reconstructive Surgery, Kindai University Faculty of Medicine, Japan
}

Received: April 09, 2018; Published: April 18, 2018

*Corresponding author: Department of Plastic and Reconstructive Surgery, Kindai University Faculty of Medicine, Osaka-sayama, Japan, Email: isogai@med.kindai.ac.jp

\section{Introduction}

In the treatment of peripheral neurological deficits, autologous nerve grafting is the first choice. The sural nerve, ante brachial cutaneous nerve, terminal branch of posterior interosseous nerve, and other choices are commonly used, however, loss of function and potential morbidity from creation of these donor sites are undesirable effects of their use. In recent years, synthetic nerve conduits and allogeneic nerve grafting materials have been introduced into clinical practice as alternatives to autologous nerve grafting [1-3]. The first attempt at nerve conduit was application for nerve regeneration using silicone tubes [4] Subsequent studies explored biodegradable materials with open internal structure as the conduit. More recently, filling the conduit with a regeneration- conducive material such as collagen demonstrated improved nerve regeneration, however, the outcome remained inferior to autologous nerve grafting and allergenic nerve transplantation [5-7]. To promote nerve regeneration, biodegradable nerve conduits need to be carefully sutured to the donor nerves at the anastomotic site as the poor adaptation often allows fibroblasts invasion into the lumen of the conduit which hampers nerve regeneration. Unfortunately, no study has elucidated the influence of diameter discrepancy between the biodegradable nerve conduit and the donor nerve for better nerve regeneration. In this study, we introduced biodegradable nerve conduits of different diameter to examine how the diameter discrepancy influences nerve regeneration.
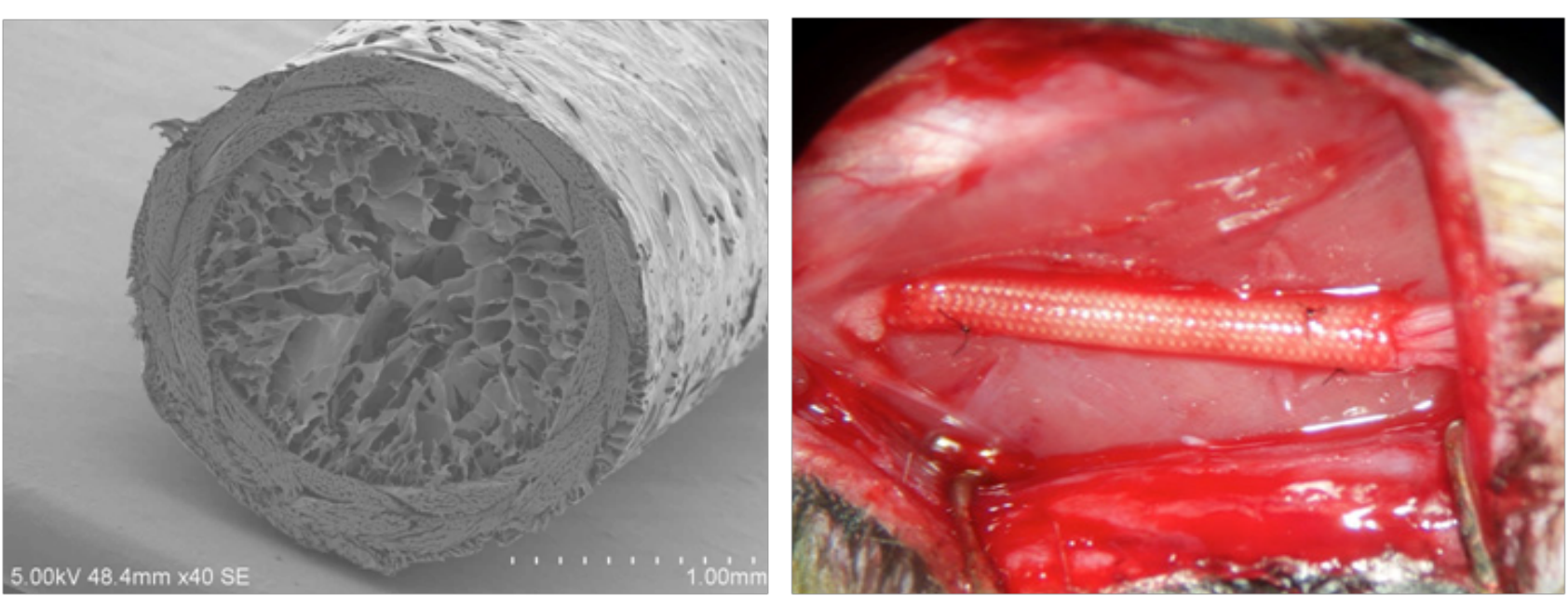

Figure 1: Scanning electron microscopic findings of biodegradable nerve conduit (A) and macroscopic finding after implantation to the sciatic nerve gap (B). 


\section{Methods}

\section{Scanning Electron Microscopy of the Biodegradable Nerve Conduit Surface}

Observation of the surface of biodegradable nerve conduit was conducted using scanning electron microscopy. The samples were dried and gold-coated with evaluation using a scanning electron microscope (S-900, Hitachi, Ltd., Japan) (Figure 1A).

\section{Surgical Placement of the Biodegradable Nerve Conduit in the Rat Sciatic Nerve}

All the animal experiments were conducted following the regulations of the Animal Experiment Committee of Kindai University. Fourteen-week-old SD rats (mean body weight 280 324 g Harlan Sprague Dawley, Indianapolis, IN, USA) were used as experimental animals ( $\mathrm{n}=18)$. Housing was maintained in a clean rack with uniform temperature $\left(22^{\circ} \mathrm{C}\right)$ and humidity (50\%) and 12-hour light-dark cycle. Radiation-sterilized ( $3 \mathrm{mG}$ ) solid food and water was provided ad libitum. After general anaesthesia with urethane and $\alpha$-chloralose, a 10 -mm gap was created by sharp transection of the sciatic nerve on one leg per rat. To fill the gap, $12 \mathrm{~mm}$ biodegradable nerve conduit was then implanted. The ends of the nerve were drawn into the open ends of the biodegradable nerve conduit by $1 \mathrm{~mm}$ on each side using a horizontal mattress stitch of 10-0 nylon suture to secure each end in place (Figure 1B). Biodegradable nerve conduits of three different diameter (1.5, 2.0, $2.5 \mathrm{~mm}$, respectively) were implanted ( $\mathrm{n}=6$ in each group). Evaluation of nerve regeneration was done 12 weeks after the surgery (Figure 2).

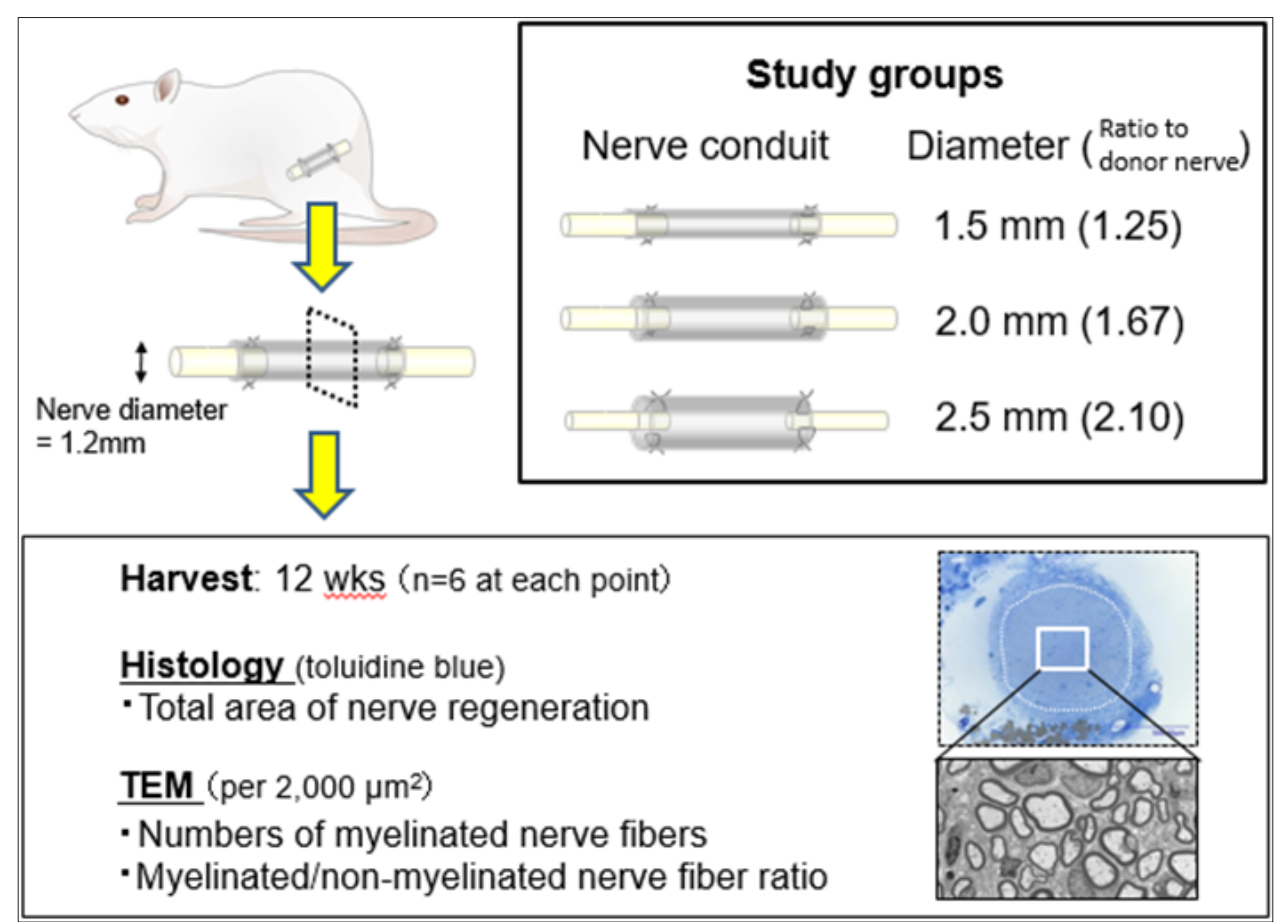

Figure 2: Experimental protocol.

\section{Evaluation of the Nerve Regeneration Using Histological Method}

At 12 weeks after implantation, the biodegradable nerve conduit was harvested and the tissues were immersion-fixed in $10 \%$ neutral buffered formalin solution for 3 days. Paraffin blocks were prepared and $5-\mu \mathrm{m}$ sections were made in the longitudinal direction with a microtome (LEICA SM2000R). Toluidine blue was used for staining area of nerve regeneration.

\section{Evaluation of Nerve Maturity Using Transmission Electron Microscopy (TEM)}

Biodegradable nerve conduits collected at 12 weeks after implantation were immersed and fixed in $4 \%$ glutaraldehyde for 3 days. The tissues were sectioned $(1.5 \mu \mathrm{m}$ thick) transversely with an ultra microtome (LEICA EM UC7) and then post-fixed with $4 \%$ osmium. The slices were dehydrated by alcohol series, stained with uranium and embedded in peon. They were sliced to ultrathin sections of $700 \mathrm{~A}$ in thicknesses, stained with urinal and lead acetate and observed using a transmission electron microscope (H-7700, Hitachi, Ltd., Japan). The sites observed was the middle region of biodegradable nerve conduit at the maximum scale possible to identify axons, $50 \mu \mathrm{m} \times 40 \mu \mathrm{m}\left(=2,000 \mu \mathrm{m}^{2}\right)$. Three visual fields near the center of each cross-section in each group were photographed, and the mean value per unit area of $2,000 \mu \mathrm{m}^{2}$ was calculated for area of militated nerve fibbers and militated/non-militated nerve fiber ratio. Image J software was used for analysis.

\section{Statistical analysis}

All experimental results were indicated as mean \pm standard deviation (SD). Statistical analyses used one-way analysis of 
variance (one-way ANOVA) and Holm post-test for comparison. Software Inc., San Diego, CA, USA), and P $<0.05$ was used as the Data analysis was conducted using GraphPad Prism (GraphPad criterion for significance.

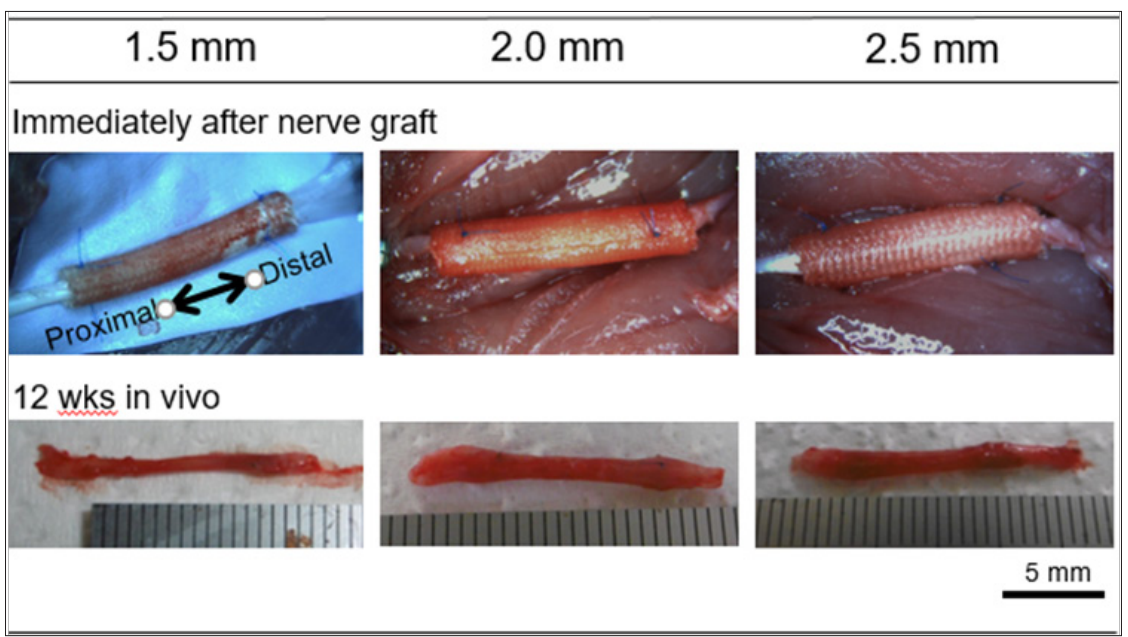

Figure 3: Macroscopic findings of biodegradable nerve conduits of different diameter.

\section{Results}

Macroscopic Finding After Implantation of the Biodegradable Nerve Conduits

Every conduit maintained a clear profile within the tissue bed with little evidence of integration with the surrounding tissue. The nerve conduits became thinner in $1.5 \mathrm{~mm}$ group at 12 weeks after implantation. In contrast, $2.0 \mathrm{~mm}$ group showed clear thickening (Figure 3).

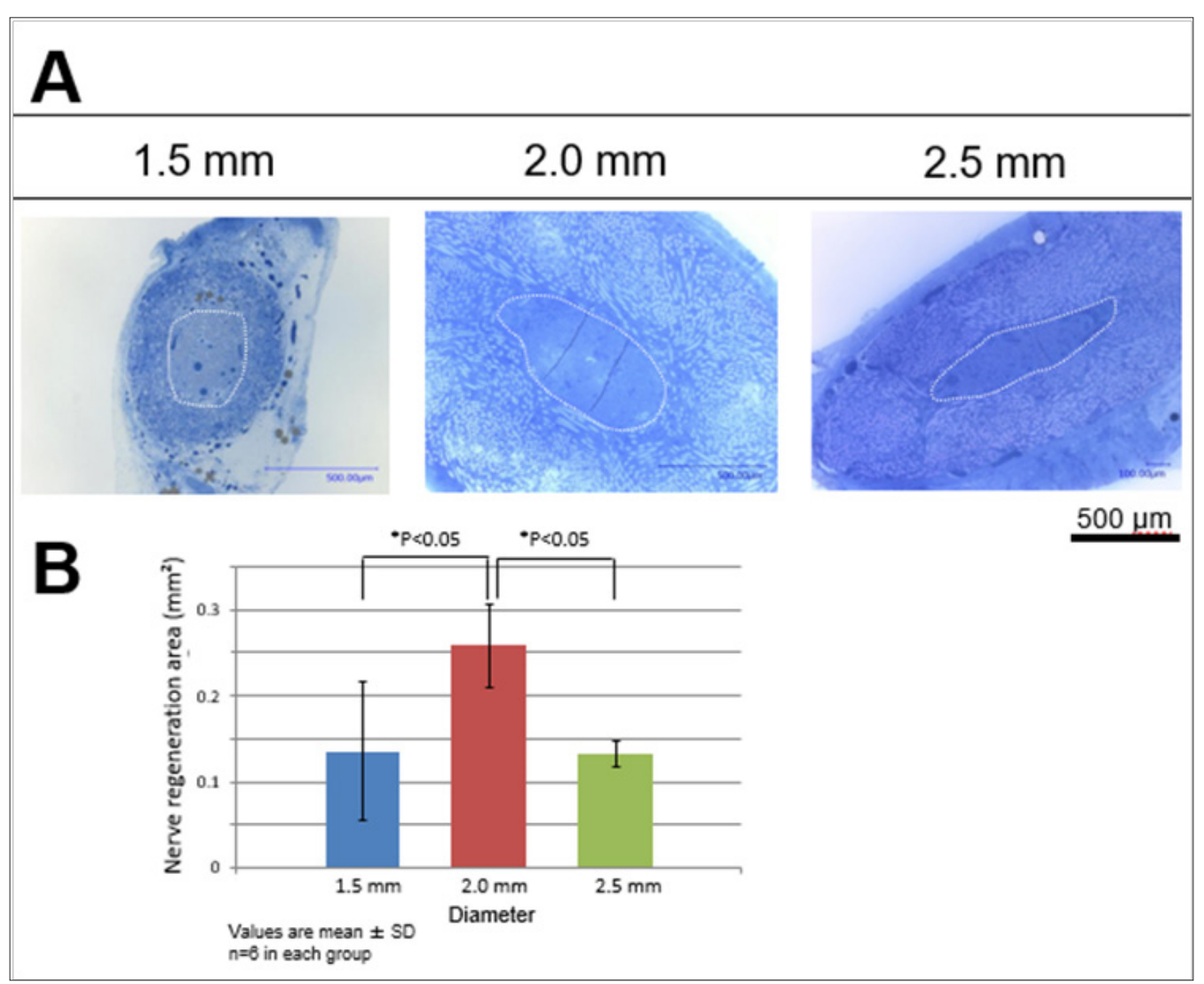

Figure 4: Histological evaluation of the biodegradable nerve conduit.

(A) The biodegradable nerve conduits implanted to the sciatic nerve gap were collected at 12 weeks in vivo. Middle region of the implanted conduit was toluidine blue-stained. (B) Area of nerve regeneration was measured. 


\section{Evaluation of Nerve Regeneration Using Histological Method}

The area of nerve regeneration in $2.0 \mathrm{~mm}$ group showed significant increase when compared with those of $1.5 \mathrm{~mm}$ and 2.5 mm group (Figure 4).

\section{Nerve Maturity at 12 Weeks Assessed by Tem}

The density of militated nerve fibbers (number of fibbers per 2000 um $^{2}$ area) in all three diameter groups was not found to differ, whereas militated/non militated nerve fibber ratio was statistically lower in the $2.5 \mathrm{~mm}$ group in comparison to $2.0 \mathrm{~mm}$ group at the respective level $(\mathrm{p}<0.05)$ (Figure 5). It was lower in $1.5 \mathrm{~mm}$ group than that of $2.0 \mathrm{~mm}$ group, though this did not achieve statistical significance.

\section{Discussion}

In the treatment of peripheral neurological deficit, the nerve conduit of hollow structure is indicated for the deficit within 2 $\mathrm{cm}$, and for the neurological deficit longer than this, allogeneic nerve transplantation and autologous nerve transplantation are indicated [1-10]. To modify the biodegradable nerve conduit is clearly needed. The nerve conduit tested in the present experiment is of the biodegradable material obtained by forming PGA fibber to a luminal structure of which the thickness of luminal wall was adjusted to about $500 \mu \mathrm{m}$. Different from the biodegradable nerve conduit having conventional hollow structure, the inner lumen is filled with collagen. It is already reported that the collagen in the inner lumen becomes the scaffold in migration and growth of various cells contributing to nerve regeneration and the nerve regenerative ability is accelerated [11-17]. Moreover, the lowmolecular-weight molecules such as albumin are able to penetrate through the biodegradable nerve conduit wall and, therefore, nerve regeneration is more excellent than the previous nerve conduit [16]. In this research, we prepared the nerve conduit prepared from biodegradable materials of different diameter to examine how the diameter discrepancy of the biodegradable nerve conduit influences nerve regeneration. As a result, it was found that, when the biodegradable nerve conduit of $2.0 \mathrm{~mm}$ diameter was implanted, the nerve regeneration and ratio of militated/non-militated nerve fibber were promoted at 12 weeks after implantation. This suggests that the optimum diameter of the biodegradable nerve conduit is about 1.5 times larger than the donor nerve to obtain better nerve regeneration.

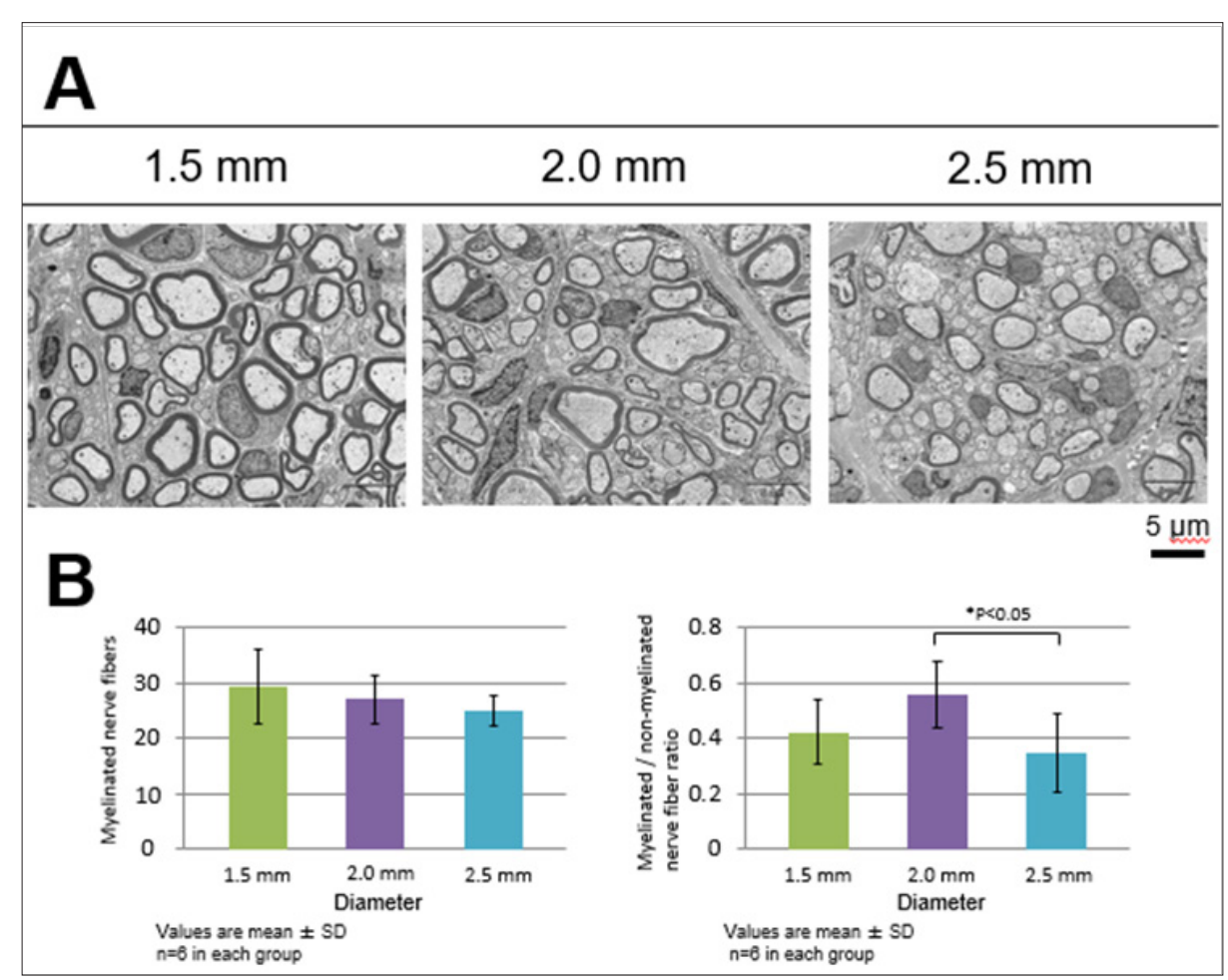

Figure 5: Transmission electron microscopic findings of the biodegradable nerve conduit.

(A) Middle region of the implanted biodegradable nerve conduit was observed at 12 weeks after implatation using transmission electron microscope. (B) The area of myelinated nerve fiber (left) and myelinated/non-myelinated nerve fiber ratio (right) per 2,000 $\mu^{2}$ were evaluated quantitatively.

\section{References}

1. Rbia N, Shin AY (2017) The Role of Nerve Graft Substitutes in Motor and Mixed Motor/Sensory Peripheral Nerve Injuries, J Hand Surg Am 42, 367-377
2. Brooks DN, et al, (2012) Processed nerve allografts for peripheral nerve reconstruction: a multicenter study of utilization and outcomes in sensory, mixed, and motor nerve reconstructions, Microsurgery 32, $1-14$ 
3. Lin MY, Manzano G, Gupta R (2013) Nerve allografts and conduits in peripheral nerve repair. Hand Clin 29: 331-348

4. Lundborg G, Hansson HA (1979) Regeneration of peripheral nerve through a performed tissue space : Preliminary observations on the reorganization of regenerating nerve fibres and perineurium. Brain Research 178: 573-576

5. Hernández-Cortés P, Garrido J, Cámara M, Ravassa FO (2010) Failed digital nerve reconstruction by foreign body reaction to Neurolac nerve conduit. Microsurgery 30: 414-416

6. Meek MF, Den Dunnen WF (2009) Porosity of the wall of a Neurolac nerve conduit hampers nerve regeneration. Microsurgery 29: 473-478

7. Moore AM, et al. (2009) Limitations of conduits in peripheral nerve repairs. Hand (NY) 4: 180-186

8. Koshima I, Harii K (1985) Experimental study of vascularized nerve conduits: multifactorial analyses of axonal regeneration of nerves transplanted into an acute burn wound. J Hand Surg Am 10: 64-72

9. Tabata Y, Nagano A, Muniruzzaman M, Ikada Y (1998) In vitro sorption and desorption of basic fibroblast growth factor from biodegradable hydrogels. Biomaterials 19: 1781-1789

10. Ducic I, Fu R, Iorio ML (2012) Innovative Treatment of Peripheral Nerve Injuries: Combined Reconstructive Concepts. Ann Plast Surg 68: 180187

11. Suzuki K, et al. (2009) Histologic and electrophysiological study of nerve regeneration using a polyglycolic acid-collagen nerve conduit filled with collagen sponge in canine model. Urology 74: 958-963
12. Matsumoto K, et al. (2000) Peripheral nerve regeneration across an 80$\mathrm{mm}$ gap bridged by a polyglycolic acid[PGA]-collagen tube filled with laminin-coated collagen fibers: a histological and electrophysiological evaluation of regenerated nerves. Brain res 868: 315-328

13. Lee JY, et al. (2012) The effect of collagen nerve conduits filled with collagen-glycosaminoglycan matrix on peripheral motor nerve regeneration in a rat model. J Bone Joint Surg Am 94: 2084-2091

14. Sahakyants T, Lee JY, Friedrich PF, Bishop AT, Shin AY (2013) Return of motor function after repair of a 3-cm gap in a rabbit peroneal nerve: a comparison of autograft, collagen conduit, and conduit filled with collagen-GAG matrix. J Bone Joint Surg Am 95: 1952-1958

15. Suzuki H, et al. (2016) Value of a novel PGA-collagen tube on recurrent laryngeal nerve regeneration in a rat model. The Laryngoscope 126: 233-239

16. Iijima Y, Ajiki T, Murayama A, Takeshita K (2016) Effect of Artificial Nerve Conduit Vascularization on Peripheral Nerve in a Necrotic Bed. Plast Reconstr Surg Glob Open 4: e665

17. Matsui Y, Yamasaki T, Inoue T, Kamba T, Ogawa O (2015) Cavernous Nerve Graft Reconstruction with a Novel Artificial Conduit during RobotAssisted Laparoscopic Radical Prostatectomy. Open Journal of Urology 5: $118-122$

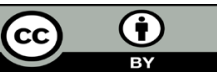

This work is licensed under Creative Commons Attribution 4.0 License

Submission Link: https://biomedres.us/submit-manuscript.php

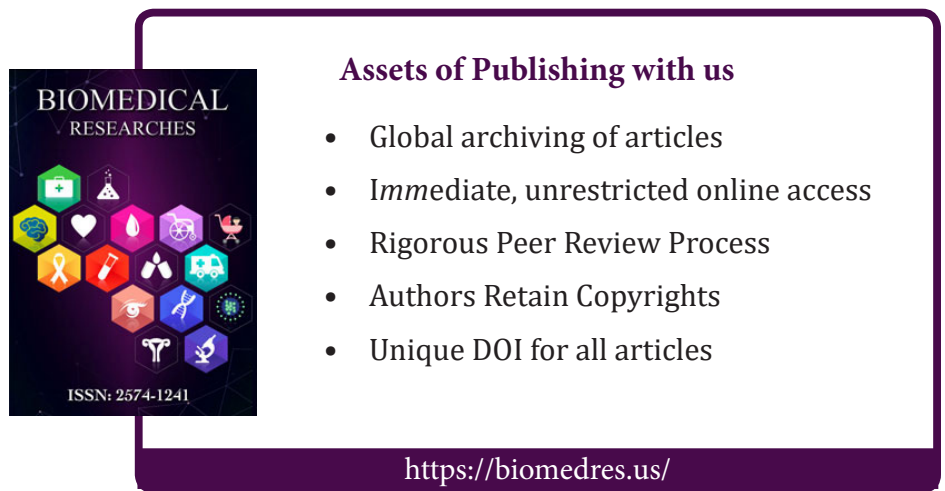

